\title{
The CASSIOPE/e-POP Suprathermal Electron Imager (SEI)
}

\author{
D.J. Knudsen ${ }^{1}$ · J.K. Burchill ${ }^{1}$ · T.G. Cameron ${ }^{1}$. \\ G.A. Enno ${ }^{1}$ - A. Howarth ${ }^{1}$ - A.W. Yau ${ }^{1}$
}

Received: 5 November 2014 / Accepted: 3 April 2015 / Published online: 19 May 2015

(C) The Author(s) 2015. This article is published with open access at Springerlink.com

\begin{abstract}
The Suprathermal Electron Imager (SEI) on the Enhanced Polar Outflow Probe (e-POP) experiment uses a microchannel-plate-intensified charge-coupled device (CCD) detector to record two-dimensional, energy-angle images of electron distributions for energies up to $350 \mathrm{eV}$. Alternatively, the SEI can be biased to measure positive ions at energies that include the ambient ionospheric population $(<1 \mathrm{eV})$ and extending to $350 \mathrm{eV}$. At the highest measurement resolution, distribution images are 64 pixels in diameter and are read out at a rate of 100 images per second. The SEI's field of view is $360^{\circ} \times \pm 4^{\circ}$, and includes viewing of the nadir and ram directions, nominally. At high latitude the nominal orientation allows coverage of most pitch angles. The SEI is included on e-POP to address the mission's principal scientific objectives, the first being to characterize polar ion outflow and its drivers including ambipolar electric fields generated by suprathermal electron populations, and direct energization of ions by plasma waves or through frictional heating. In addition, the SEI's focus on low energies and high time resolution allows a unique view of suprathermal particle populations and their role in wave-particle interactions, in support of e-POP's second scientific objective: to study plasma waves and wave propagation in the high-latitude ionosphere. Observations taken within geophysically quiet regions indicate that the instrument can track bulk ion flow velocity with a resolution of order $25 \mathrm{~m} / \mathrm{s}$ or better.
\end{abstract}

Keywords Space instrumentation · Charged particle detectors · Ionospheric measurements

\section{Introduction}

The Enhanced Polar Outflow Probe (e-POP) scientific payload is part of the CASSIOPE satellite. CASSIOPE was launched from Vandenberg Air Force Base on September 29, 2013 into a polar elliptical orbit having an inclination of $81^{\circ}$, an apogee of $1500 \mathrm{~km}$, and perigee of $325 \mathrm{~km}$. e-POP was designed with three scientific objectives in mind, namely to investigate

D.J. Knudsen

knudsen@ucalgary.ca

1 Department of Physics and Astronomy, University of Calgary, 2500 University Drive NW,

Calgary T2N $1 \mathrm{~N} 4, \mathrm{AB}$, Canada 
the properties and causes of polar ion outflow, to study plasma waves and wave propagation in the ionosphere, and to search for neutral upwelling. See Yau and James (2015, this issue) for further discussion of these objectives, and of the CASSIOPE/e-POP mission overall.

Research over the past decades has identified several candidate drivers of polar outflow (e.g. see Strangeway et al. 2005), including direct heating of ions either by plasma waves or through frictional heating and subsequent upflow due to the mirror force, and ambipolar outflow driven either by photoelectrons or by heated electron populations resulting from soft auroral precipitation. While direct heating of ions by waves near the ion cyclotron frequency is responsible for the most intense heating events in the topside ionosphere (André et al. 1998; Knudsen et al. 1998; Lynch et al. 1999), photoelectron-driven ambipolar outflow is more widespread and persistent (Abe et al. 1993). Photoelectrons of atmospheric origin extend to energies as high as $800 \mathrm{eV}$, though the bulk of the distribution is at much lower energies. Photoelectron intensities can vary dramatically in response to changes in solar EUV irradiation (Peterson et al. 2008); these modulations presumably cause commensurate variations of ion outflow as well, although such a link has not yet been demonstrated experimentally. The efficiency of resonant charge exchange between neutral and ionized atomic oxygen or hydrogen (i.e. between $\mathrm{O}$ and $\mathrm{O}^{+}$and between $\mathrm{H}$ and $\mathrm{H}^{+}$) and of the accidentally resonant $\mathrm{O}-\mathrm{H}$ charge exchange (between $\mathrm{H}_{\text {and }} \mathrm{O}^{+}$and $\mathrm{O}$ and $\mathrm{H}^{+}$) suggests that ion outflows are accompanied by a neutral component as well (Fuselier et al. 2001).

e-POP is designed to observe the key parameters relevant to outflow and its drivers, and therefore includes a magnetic field instrument (MGF) to characterize field-aligned current systems (Wallis et al. 2014), a plasma wave receiver (RRI), an auroral imager (FAI) to provide auroral context (Cogger et al. 2014), a neutral mass spectrometer (NMS) to characterize neutral flows, a low-energy ion mass spectrometer (IRM) (Yau et al. 2014), and the suprathermal electron imager (SEI) which is the subject of this paper.

The term "suprathermal" refers to energies above those of the ionospheric core population (less than $1 \mathrm{eV}$, typically), but less than those typical of the magnetosphere, for example the $\sim 1 \mathrm{keV}$ temperatures characteristic of the central plasma sheet. Suprathermal energies are key to the process of ambipolar outflow, wherein elevated electron temperatures enhance electron diffusion and cause an electric field that counteracts the gravitational force on the ions. Conservation of energy requires that, in order for ions to escape, the electron thermal energy must exceed the gravitational potential energy of ions, which is $10 \mathrm{eV}$ in the case of $\mathrm{O}^{+}$. The SEI measures electron intensities at energies from near the plasma potential to $350 \mathrm{eV}$, which is the energy range most relevant to ion outflow driven by ambipolar electric fields. The SEI can also be configured to measure positive ions, also to energies of up to $350 \mathrm{eV}$.

CASSIOPE's orbital velocity is $6.9 \mathrm{~km} / \mathrm{s}$ at apogee, and $8.0 \mathrm{~km} / \mathrm{s}$ at perigee. At 100 images per second, the SEI is capable of resolving structures as small as $69 \mathrm{~m}$ at apogee (80 $\mathrm{m}$ at perigee), comparable to the smallest structures $(\sim 100 \mathrm{~m})$ associated with core plasma energization regions in the topside ionosphere (e.g. Knudsen et al. 1994).

While ion outflow is not expected to be as highly structured as the soft electron precipitation that sometimes drives it, it is important to characterize the degree of structure within electron drivers both in order to understand the role of structured energization on outflow, and to provide clues as to the source of the suprathermal electron beams themselves. For example, dispersive Alfvén waves with scales of the order of $1 \mathrm{~km}$ transverse to the geomagnetic field $\mathbf{B}$ are known to be associated with transient bursts of suprathermal electrons (e.g. Stasiewicz et al. 2000).

The SEI's rapid image rate is possible in part due to a hemispherical focusing system that is able to image in both energy and arrival angle. This is in contrast to the much more 
common top-hat analyzer (Carlson et al. 1982), which must step through energy with time, though rapid sampling of distributions was made possible on the FAST satellite through the use of multiple top-hat analyzers (Carlson et al. 1998). Furthermore, for a given interhemispherical potential $\Delta V$, the top-hat admits particles having energies of several times $q \Delta V$, whereas the SEI selects for particle energies of the order of $q \Delta V / 2$ and below, making it better suited to the study of low-energy particle populations. However, it should be noted that a miniaturized version of the top-hat analyzer has been used successfully to observe thermal (core) electron populations (Pollock et al. 1996), as have other techniques (e.g. MacDonald et al. 2006).

The SEI's energy-angle imaging scheme was first used in orbit with the Freja Cold Plasma Analyzer (Whalen et al. 1994), the detector for which consisted of a sparse array $(16 \times 16)$ of small, discrete anode pixels arranged in columns and rings and connected to a network of electronic charge amplifiers. This scheme was adequate for the study of strong ion heating in the topside ionosphere (Knudsen et al. 1998; Knudsen and Wahlund 1998), but lacked the ability to resolve most plasma flows. For example, a cross-track plasma flow velocity of $100 \mathrm{~m} / \mathrm{s}$ manifests as an angular deviation of less than $1^{\circ}$ as seen from a spacecraft moving at $8 \mathrm{~km} / \mathrm{s}$ in low earth orbit, whereas the CPA's sixteen radial spokes were separated by $18^{\circ}$. In order to resolve small deviations in angle (and also small changes in ram energy) caused by typical plasma ionospheric plasma flows, the SEI uses an improved detector scheme based on charge-coupled device (CCD) technology, providing full coverage of the detector plane with 3000 pixels in order to exploit information from all particles reaching the detector surface (Knudsen et al. 2003).

Prior to the launch of e-POP, the ability of the ion-detecting version of the SEI (the SII) to track rapid changes in ion velocity and temperature was tested and demonstrated on several sub-orbital sounding rocket flights. Using data from the GEODESIC sounding rocket, Burchill et al. (2004) used the SII to resolve strongly heated ion populations confined within filamentary density cavities only $20 \mathrm{~m}$ in diameter, and to track ion flow velocity variations within a $3 \mathrm{~Hz}$ shear Alfvén wave. Burchill et al. (2010) used SII measurements from the Cusp 2002 rocket in the low-altitude dayside cusp to resolve ion upflow velocities of the order of $100 \mathrm{~m} / \mathrm{s}$. And Sangalli et al. (2009) and Burchill et al. (2012) compared ion velocities measured during JOULE-II rocket experiment with E-cross-B and neutral wind velocities and demonstrated measurement accuracies of better than $20 \mathrm{~m} / \mathrm{s} \mathrm{r.m.s.}$

In this paper we provide a technical description of the e-POP SEI instrument in Sect. 2, followed in Sect. 3 by data examples taken during pre-launch calibration and in flight.

\section{Instrument Description}

Figure 1 shows the mounting position and orientation of the SEI sensor on CASSIOPE. The spacecraft uses momentum wheels for three-axis stabilization which allows its orientation to be set arbitrarily, however for most operations the satellite's $+X$ axis is parallel to the orbital velocity vector, and the $+\mathrm{Z}$ axis is nadir-pointing. Figure 2 is a photograph of the SEI instrument, which includes a cylindrical sensor head mounted on a $43 \mathrm{~cm}$ boom, fiber-optic and electrical cables, and an electronics unit (EU). Table 1 lists physical dimensions and mass properties of these components.

Figure 3 shows the principle of operation of the SEI instrument. In this view a beam of charged particles having a range of energies is directed from right to left into a circular aperature near the top of the SEI sensor head. An entrance aperture extends $360^{\circ}$ around the sensor perimeter, minus obstructions due to three internal support posts, each of which 
Fig. 1 The SEI on CASSIOPE.

The SEI field of view, highlighted by the shaded region, includes particle velocities within $\pm 4^{\circ}$ of the entrance aperture plane. The spacecraft $X$ and $Z$ axes are oriented in the ram and nadir directions, nominally, though orientation can be set arbitrarily

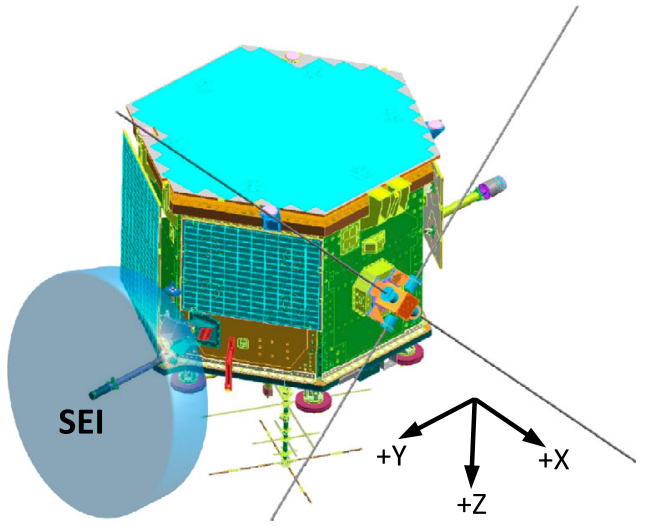

Fig. 2 The e-POP SEI sensor and boom assembly, electrical and fiber optic cables, and electronics unit (EU). Photo: E. Peter King

Table 1 e-POP SEI physical characteristics

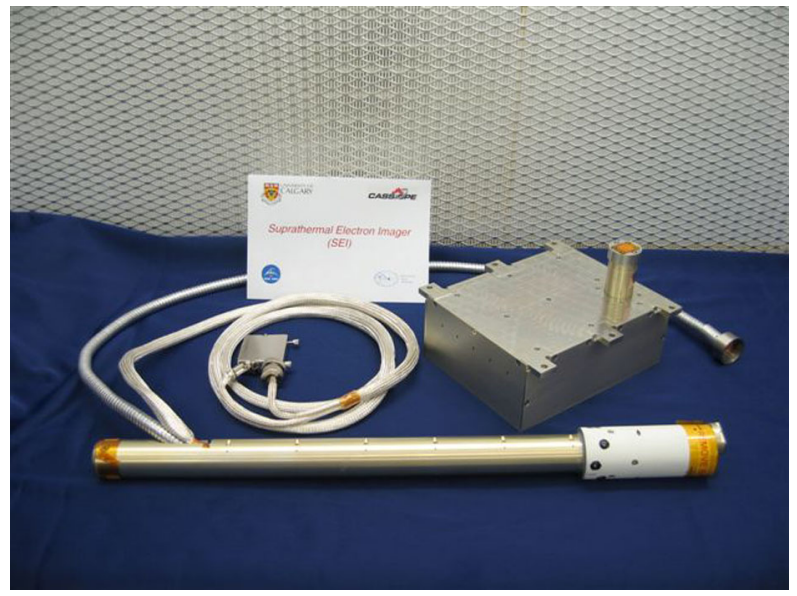

\begin{tabular}{ll}
\hline Parameter & Value \\
\hline Mass, Sensor and Cables & $2.8 \mathrm{~kg}$ \\
EU Mass & $2.4 \mathrm{~kg}$ \\
Sensor length & $12.2 \mathrm{~cm}$ \\
Sensor diameter & $4.6 \mathrm{~cm}$ \\
Boom length & $43 \mathrm{~cm}$ \\
EU dimensions & $20.3 \times 18.5 \times 7.8 \mathrm{~cm}$ \\
\hline
\end{tabular}

subtends an angle of approximately $10^{\circ}$ as seen from the center of the detector. Directly inside the exterior aperture is a field-free region bounded by two flat, annular electrodes which collimate the incident beam to within $\pm 4^{\circ}$ of the entrance plane. After traversing this region, particles encounter a region in which a radial electric field is supported by two hemispherical electrodes, the inner containing a gridded window. Inside the inner hemisphere is a field-free region bounded on the bottom by a flat mesh grid. The combined radial and field-free regions disperse particles according to their incident energy per charge, with higher-energy particles landing closer to the edge of the circular detector region. The inner-hemisphere 

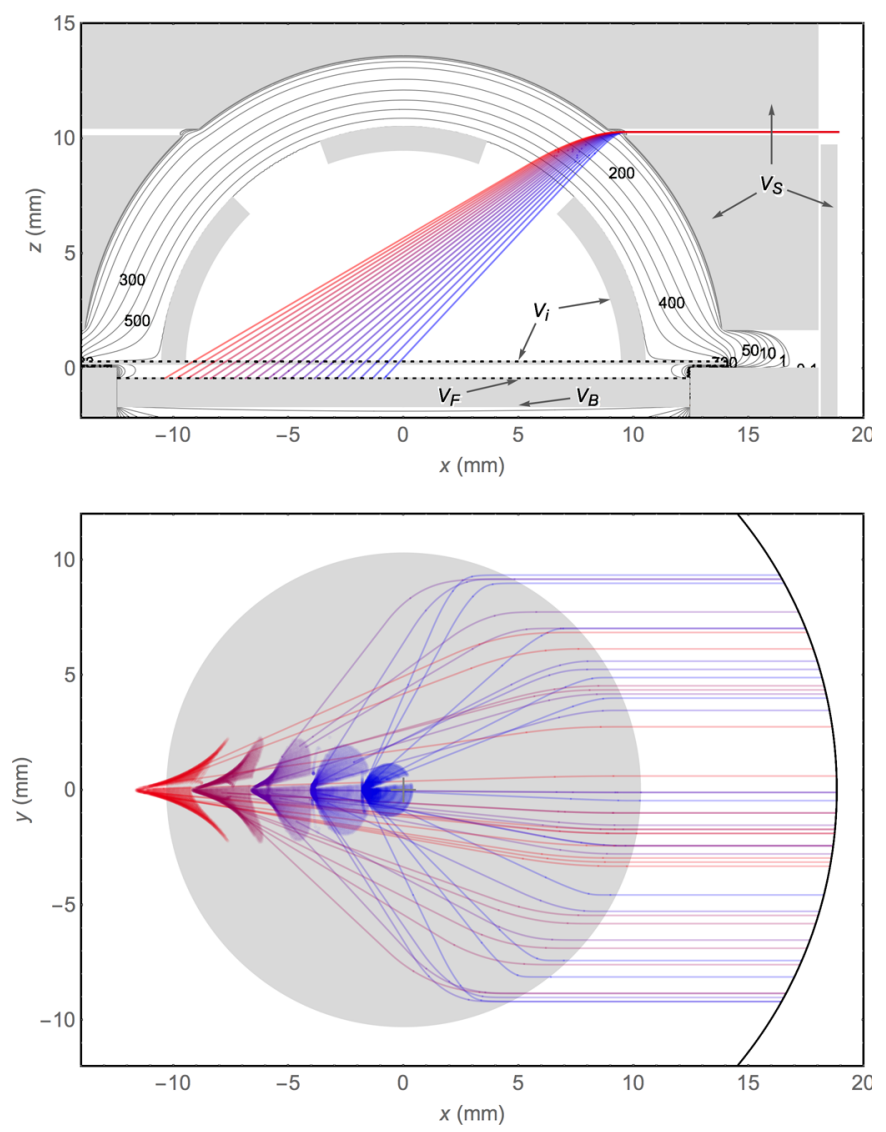

Fig. 3 SEI imaging principle. A radial electric field between hemispherical electrodes deflects particles onto the detector plane according to their incident energy, and focuses in detector azimuth according to their angle of incidence. This example uses an external skin voltage $-5.3 \mathrm{~V}$ and a deflection voltage $V_{i}$ of $+708 \mathrm{~V}$. The top panel shows trajectories of electrons spanning an energy range of 9.6 to $284 \mathrm{~V}$ as they traverse equipotential lines (shown in gray). The bottom panel (top view) shows unidirectional beams having energies (increasing from blue to red) of 10, 51, 116, 194, and $284 \mathrm{~V}$

voltage $V_{i}$ determines the upper energy limit of the detector, meaning the energy of particles landing at the outer radius. Laboratory measurements (see Sect. 3) show the upper energy to be approximately $q V_{i} / 2$.

The lower panel of Fig. 3 gives a top view of the detector and shows the ideal "pointspread function" of unidirectional particle beams distributed uniformly across the entrance aperture for a set of five different energies. Polar angle on the detector maps to angle of incidence for particle arriving in the plane of the detector and aperture.

Table 2 summarizes the control voltages (see Fig. 3) which determine the focusing and detection properties of the SEI sensor. The exterior skin is coated with a conductive, thermalcontrol material and is biased to a voltage $V_{s}$ relative to the spacecraft and boom. The sensor cap is biased at this same voltage. When measuring electrons, a negative bias of a few Volts (relative to spacecraft ground) repels the relatively high-density thermal (or core) electrons which would otherwise saturate the detector, the gain of which is set to observe the lower-density, suprathermal population. With positive ions, the negative bias compensates 
Table 2 SEI sensor voltage ranges

\begin{tabular}{llll}
\hline Parameter & Range $(\mathrm{V})$ & $\begin{array}{l}\text { Electron Mode } \\
\text { Typical }(\mathrm{V})\end{array}$ & $\begin{array}{l}\text { Ion Mode } \\
\text { Typical (V) }\end{array}$ \\
\hline$V_{S}$ & {$[0$ to -30$]$ or $[0$ to +3$]$} & -2 to -5 & -2 \\
$V_{i}$ & {$[0$ to -1000$]$ or $[0$ to +1000$]$} & +500 & -100 \\
$V_{F}$ & {$[0$ to -2400$]$ or $[0$ to +1000$]$} & +500 & -1600 \\
$V_{B}$ & {$[0$ to +3400$]$ or $[0$ to +1400$]$} & +2100 & 0 \\
$V_{P}$ & {$[0$ to +5000$]$ or $[0$ to +7000$]$} & +5000 & +5000
\end{tabular}

for possible positive excursions in spacecraft potential which could otherwise repel the entire thermal ion population.

e-POP has no means to determine the spacecraft floating potential on-board, and there is no capability to control $V_{s}$ actively. Rather, $V_{s}$ is set to a fixed negative value during each period of operation, typically in the range -2 to $-5 \mathrm{~V}$ in order to account for expected values of spacecraft potential, which are expected to remain within 1 or $2 \mathrm{~V}$ of the plasma potential in most situations. Uncertainty in the actual value of the spacecraft-to-plasma potential must be kept in mind while interpreting SEI data. The SEI design allows for the sensor skin to be biased as low as $-30 \mathrm{~V}$ to exclude the lower-energy range of the daytime photoelectron spectrum, allowing the higher-energy portion to be recorded with higher sensitivity.

Figure 4 is a schematic of the SEI sensor head showing the elements of the focusing system described above, and of the detector system that follows. Once particles pass through the flat grid bounding the field-free hemispherical region, they are accelerated onto a pair of micro-channel plates (MCPs) via an accelerating potential $V_{F}-V_{i}$ (see Fig. 3). The need for efficient triggering of the MCP requires $V_{F}$ values of at least several hundred volts (positive) for electron detection. For ion detection $V_{F}$ is set to values of between -1500 to $-1800 \mathrm{~V}$, typically.

The MCP gain (average number of output electrons per MCP firing) is determined by the difference potential $\Delta V=V_{B}-V_{F}$. For the SEI, which uses a pair of MCPs, individual particle events can easily be seen for values of $\Delta V$ of the order of $1800 \mathrm{~V}$. However, much lower gain values are used with the SEI due to the fact that the detector operates in so-called analog mode, meaning the sampled output of the CCD represents an analog integration of many particle events contributing to each pixel.

In contrast, pulse-counting detectors require an MCP gain large enough that each particle event can be detected by an electronic amplifier. This tends to shorten their operational lifetime relative to analog-mode detectors since MCP lifetime is a function of total extracted charge. Analog-mode detectors also allow for particle event rates that are many orders of magnitude higher than can be achieved with particle-counting detectors, since the latter are limited by the need to allow electronic circuits to recover between individual MCP firings. See Eberhart (1981) for a general overview of MCP operation.

Behind the MCP is a phosphor screen. The MCP-phosphor combination is most commonly used in conjunction with a photocathode material for optical image intensifiers or UV-imaging detectors (e.g. Anger et al. 1987; Cogger et al. 1995). In these photon detectors, the phosphor screen is coated with a thin layer of aluminum, in part to prevent optical feedback to the photocathode. This layer is not necessary for charged-particle detection, and in fact is undesirable in that the layer absorbs electron energy and therefore reduces phosphor output for a given phosphor potential. For the SEI application, a transparent conducting layer of indium-tin oxide is deposited onto the input end of the fiber-optic taper, on top of 
Fig. 4 Schematic of the SEI sensor and key components. Image: I. Wevers

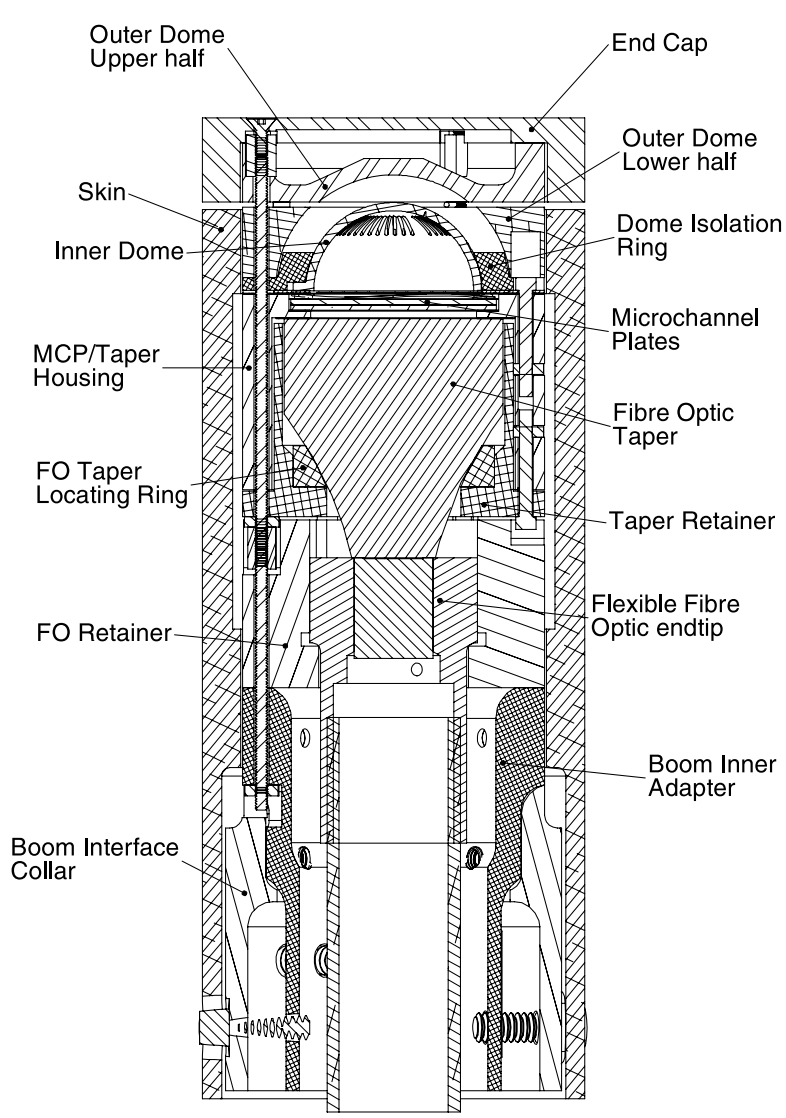

which the phosphor grains are deposited. Aase et al. (2011) describe tradeoffs relating to phosphor screen selection and operation, including that of the particular screen (P43 material) used for the SEI, chosen for a reasonable combination of rapid decay rate and efficiency (photons per incident electron and energy).

The visible image created on the phosphor screen inside the SEI sensor is transferred to the SEI's power and electronics unit (EU) through a coherent fiber-optic bundle. A fiber optic taper is used to match a commonly-available MCP diameter $(2.5 \mathrm{~cm})$ to the sub-cm width of input window of the CCD used in this application, and to reduce the diameter of the coherent fiber bundle needed to connect them. The EU houses the CCD and associated read-out electronics, a digital signal processor-based instrument controller board, and low and high-voltage power-supply boards. The basic readout rate is 100 frames per second.

The SEI uses a model CCD67 manufactured by E2V Technologies. This CCD is a frametransfer device, meaning exposed images are shifted rapidly to a storage area where they are read out while the next image is accumulated. The active image area comprises $268 \times$ 264 pixels, each $26 \times 26 \mu \mathrm{m}$. The relatively large pixel format is well-suited to the SEI application, which requires high read-out rates but not high pixel resolution (relative to optical applications). Due to the rapid frame rate of the SEI, little dark signal accumulates at nominal operating temperatures $\left(20-30{ }^{\circ} \mathrm{C}\right)$, and no active cooling is required. Pixels are binned $4 \times 4$ on chip; output is sampled at 8 bits and cropped in software to a circular-shaped 
Table 3 SEI data-taking modes

Table 4 SEI performance characteristics

\begin{tabular}{lllll}
\hline Mode & $\begin{array}{l}\text { Image rate } \\
\left(\mathrm{s}^{-1}\right)\end{array}$ & $\begin{array}{l}\text { Image dia. } \\
(\text { pixels })\end{array}$ & $\begin{array}{l}\text { Data rate } \\
(\mathrm{kbits} / \mathrm{s})\end{array}$ & Comment \\
\hline Housekeeping & 0 & 0 & 6.8 & \\
Analog & 0 & 0 & 13.2 & \\
Normal & 100 & 32 & 651 & \\
Hi-res & 100 & 64 & 2500 & \\
Integration & $100 / N$ & 32 & $16.4-631$ & $N=2^{[1-5]}$ \\
\hline
\end{tabular}

\begin{tabular}{|c|c|c|}
\hline Parameter & Value & Comment \\
\hline Pixel resolution & 64 or 32 pixels & Image diameter \\
\hline Angular resolution & $9^{\circ}$ & PSF FWHM \\
\hline Energy resolution & $20 \%$ & PSF FWHM \\
\hline Ion velocity resolution & $25 \mathrm{~m} / \mathrm{s}$ & \\
\hline Time resolution & $10 \mathrm{~ms}$ & per image \\
\hline Geometric factor & $7 \times 10^{-4} \mathrm{~cm}^{2} \mathrm{sreV}$ & per pixel \\
\hline Energy range & $0-350 \mathrm{eV}$ & adjustable \\
\hline Field of view & $360 \times \pm 4^{\circ}$ & see Fig. 1 \\
\hline
\end{tabular}

region 64 pixels in diameter, along with additional summing within the instrument controller depending on instrument mode.

The SEI instrument electronics unit (EU) provides all power supplies and readout circuitry in support of the CCD which is used to create the SEI science data. The EU consists of low- and high-voltage power converters, a Motorola DSP56309-based digital controller, and a high-speed, low-noise CCD readout subsystem. The EU is installed inside the spacecraft on the e-POP deckplate. Two umbilicals run between the EU and the SEI sensor, one being an electrical cable carrying high voltages to the sensor and the second a flexible, coherent fibre-optic cable which transfers the visible phosphor image to the CCD. In other words there are no electrical data connections between the EU and sensor; all scientific information is contained in the visible images carried by the fiber-optic cable.

Table 3 summarizes the SEI's data-taking modes. Housekeeping modes provide engineering data only and will not be discussed here. Science-taking modes require CCD imaging. The basic science imaging mode (Normal mode) reduces the sampled image diameter from 64 to 32 square pixels by binning in software; Hi-res mode retains the full, 64-pixel-diameter resolution. Both modes use the basic image rate of 100 per second. Integration mode conserves telemetry bandwidth through software binning both in pixel space, to 32 pixels in diameter, and in time, up to $640 \mathrm{~ms}$ (64 images).

\section{Instrument Performance}

The ideal performance of the SEI's electrostatic focusing system is described in Burchill (2003) and Knudsen et al. (2003), which we summarize briefly below and in Table 4. Particle focusing performance can be characterized by the instrument's point-spread function (PSF), a term borrowed from optical imaging to represent the response to a spatially uniform, collimated input beam (or equivalently, a beam resulting from a distant point source.) 
Fig. 5 Image of a $200 \mathrm{eV}$ electron beam during pre-launch laboratory testing with a deflection voltage $V_{i}=+1000 \mathrm{~V}$. Black circles indicate positions of internal support posts

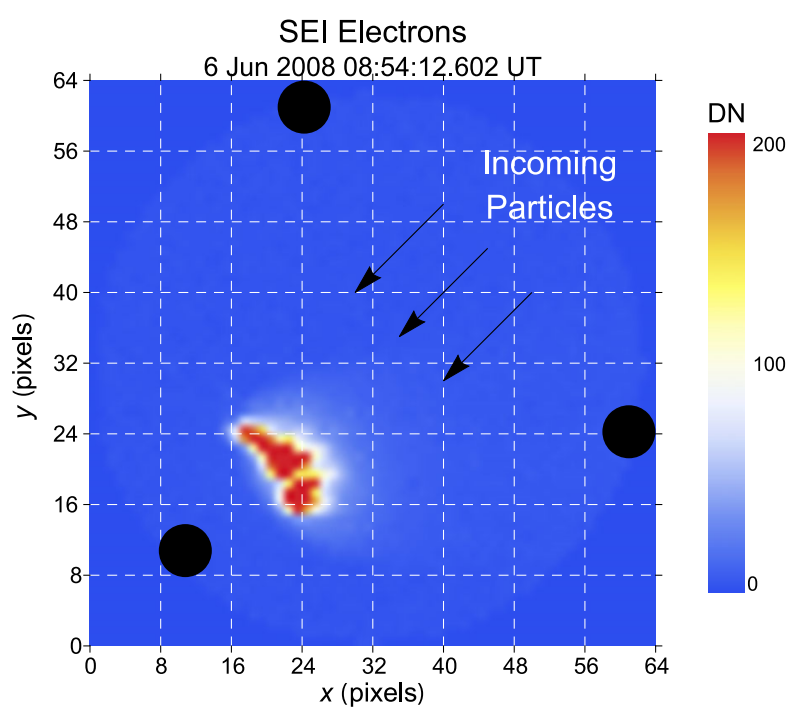

For application to the SEI, the PSF is the response to a mono-energetic, unidirectional particle beam distributed uniformly across the entrance aperture.

The response to a finite-temperature beam can be calculated as the convolution of the incident distribution with the PSF. In practice this is done by using an appropriate distribution as input to a Monte-Carlo simulation, which in addition to simplicity brings the advantage of predicting statistical noise resulting from finite particle numbers. In most circumstances, counting statistics dominates other sources of noise such as MCP pulse-height variability and electronic read-out noise (see Knudsen et al. 2003). Figure 3 shows a set of ideal PSFs from a numerical model; Fig. 5 shows an image of a $60 \mathrm{eV}$ electron beam observed in the laboratory, which approximates the actual PSF as the laboratory beam is highly collimated. However, the measured distribution clearly exhibits structure that does not appear in the simulation. We attribute this to spatial nonuniformity of the test beam, although interaction with support posts inside the sensor may also contribute.

Another measure of instrument performance is the geometric factor, which relates the differential flux of an incident beam to the count rate of a single pixel. In the case of the SEI in Hi-res mode, the geometric factor is approximately $7 \times 10^{-4} \mathrm{~cm}^{2} \mathrm{sreV}$ (Burchill 2003). Simulations of the SEI show that count rate in a single pixel is proportional to differential particle flux, as opposed to differential energy flux, which is the more usual case. This relates to the fact that the shape of the PSF depends on energy, which in turn affects the number of pixels sampling each distribution. The PSF size and geometric factor depend strongly on the accelerating potential between the sensor skin and plasma (Knudsen et al. 2003).

The SEI's resolution in azimuthal angle is determined by the angular size of the PSF, approximately $9^{\circ}$ full-width at half maximum (FWHM) in the case where the sensor potential is equal to the plasma potential. The ideal energy resolution $\Delta E / E$ of the SEI is approximately $20 \%$, determined from the radial size of the PSF along with the so-called energy-radius curve that relates the two quantities. Figure 6 shows the energy-radius curve as measured during laboratory testing prior to launch.

The $20 \%$ energy resolution figure specifies the SEI's ability to resolve distinct features in the energy spectrum of incident particles; the interpretation of the $9^{\circ}$ angular resolution is similar. The instrument's ability to track and resolve ion flows is much better, however, 


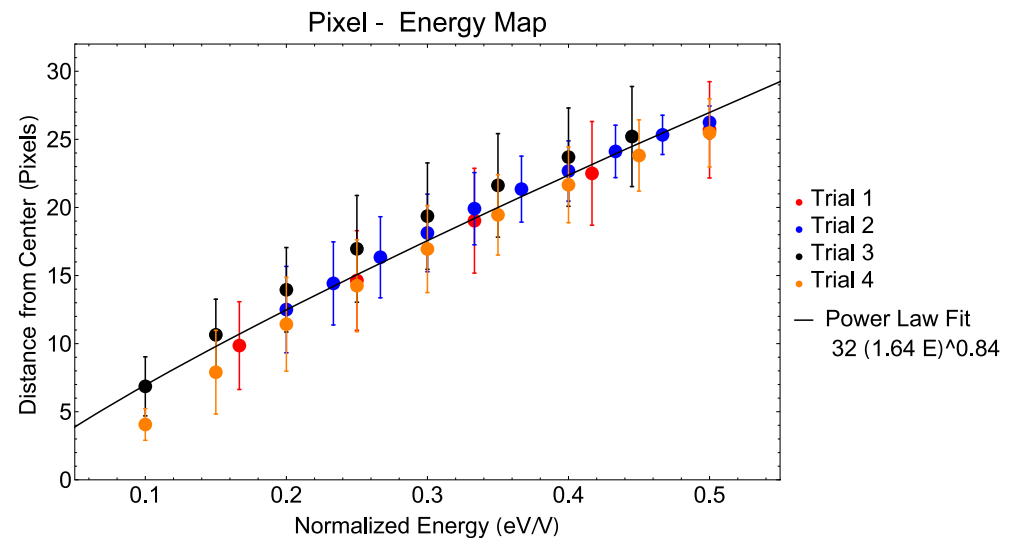

Fig. 6 Electron beam energy normalized to $V_{i}$ versus signal centroid distance from detector center for four different detector sectors as measured during laboratory testing. Vertical bars show the standard deviation of the signal distribution in radius

since in that case the objective is not to resolve distinct features, but rather to track small deviations in angle and energy caused by geophysical plasma flows superimposed on the ram velocity (negative of the satellite velocity). In this mode of operation, the average ion velocity can be resolved to high precision through a centroid calculation, as long as the detector's pixel density is high enough to resolve the rammed ion distribution with multiple pixels.

Numerical simulation of the SEI in ion mode shows that systematic errors in ion velocity due to pixelation are less than $1 \mathrm{~m} / \mathrm{s}$ in Hi-res mode (64-pixel diameter image). These errors remain of the order of $1 \mathrm{~m} / \mathrm{s}$ for Normal mode (32 pixels); they rise to $\approx 20 \mathrm{~m} / \mathrm{s}$ for a hypothetical image diameter of 16 pixels, and to several hundred $\mathrm{m} / \mathrm{s}$ for an 8 -pixel-diameter detector, assuming large pixels that cover the detector plane completely. Particle counting noise will add to these errors.

Figure 7 shows SEI ion-mode observations of a rammed ion population at an altitude of $1049 \mathrm{~km}$ in the sunlit polar cap. Comparison with the e-POP IRM instrument indicates that these ions are predominantly $\mathrm{O}^{+}$. The $\mathrm{O}^{+}$ram energy of $\sim 5 \mathrm{eV}$ plus the exterior skin bias $V_{s}$ of $-2.3 \mathrm{~V}$ places the ion signal well away from the detector center. For fixed spacecraft attitude, the position of the rammed ion signal is relatively stationary on the detector due to the dominance of satellite velocity over nominal plasma drifts. However, small deviations indicative of plasma flows are evident in Fig. 8, which shows the first moment (centroid) in pixel coordinates as a function of time. These fluctuations correspond to velocity variations of several hundred $\mathrm{m} / \mathrm{s}$, at times exceeding $1 \mathrm{~km} / \mathrm{s}$. Scientific interpretation of these results in a geophysical context is left to future publications, however structured flows of this magnitude are typical of the polar cap (e.g. Bahcivan et al. 2013).

First-moment fluctuations shown in Fig. 8 are the sum of both geophysical flows and instrument noise (dominated by particle counting statistics as mentioned above.) The instrument's noise level, and therefore its velocity resolution has been estimated by surveying data from quieter, sub-auroral regions (not shown), resulting in fluctuation levels as low as $0.2^{\circ}$ (not shown). This value provides an upper limit to the cross-track ion velocity resolution $(1 \sigma)$ of approximately $25 \mathrm{~m} / \mathrm{s}$ for a ram velocity of $7000 \mathrm{~m} / \mathrm{s}$. It is worth noting that with the exception of possible degradation by internal support posts, SEI measurement performance 
Fig. 7 One-second average of 100 images of rammed ions at an altitude of $1049 \mathrm{~km}$ in the sunlit polar cap. Detector energy increases outward radially to a maximum of $25 \mathrm{eV}$. Sensor skin voltage $V_{S}$ is $-2.3 \mathrm{~V}$. The red arrow shows the magnetic field direction; the blue arrow is opposite the spacecraft velocity. Signal extending to the right in the image is due to smearing in the $\mathrm{CCD}$ readout direction
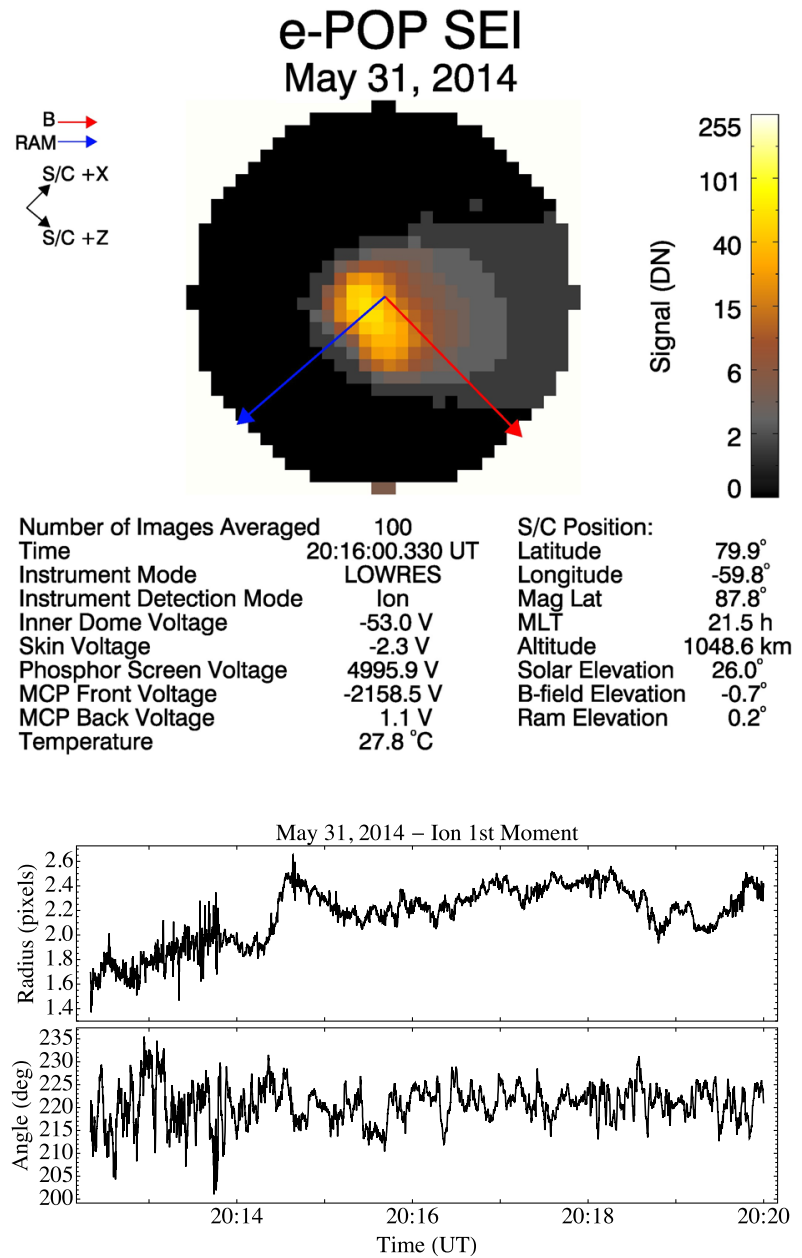

May 31,2014 - Ion 1st Moment
Fig. 8 Time series of ion signal first moments in the sunlit polar cap (same event as Fig. 7) in polar coordinates relative to the detector center, and with angle measured counter-clockwise from the right. An angular deviation of $1^{\circ}$ corresponds to a cross-track velocity variation of approximately $120 \mathrm{~m} / \mathrm{s}$ is the same for all angles of incidence due to the full and uniform coverage of the detector plane by the CCD detector.

Figure 9 shows an example of an electron image taken within a region of patchy pulsating aurora (PPA) as determined by images (not shown) from a THEMIS ground-based all-sky camera operating in Fort Yukon, Alaska (Donovan et al. 2006). The distribution is broad in energy out to the $350 \mathrm{eV}$ outer limit of the detector, and also in angle. Figure 10 shows spectrograms of energy and detector angle (linearly related to pitch angle) versus time over a $10 \mathrm{~s}$ period, illustrating the temporal variability of the fluxes at periods characteristic of PPA.

A broad angular distribution over the up-going hemisphere is expected of electrons backscattered from the keV-magnitude primaries associated with patchy pulsating aurora. The fact that the down-going hemisphere is also populated indicates that the primary incident beam extends down to suprathermal energies, or that up-going electrons are reflected back down from an upward electric field above the satellite. This is a topic for future study.

While the relation between differential particle flux and particle count rate at the MCP input can be determined through simulation, the intensity of the resulting signal at the CCD 
Fig. 9 One-second average of 100 electron-mode images taken at an altitude of $369 \mathrm{~km}$ within a region of patchy pulsating aurora. Energy ranges from $5 \mathrm{eV}$ at the center to $350 \mathrm{eV}$ at the perimeter. Red and blue arrows show directions of magnetic field and the negative of the satellite velocity, respectively

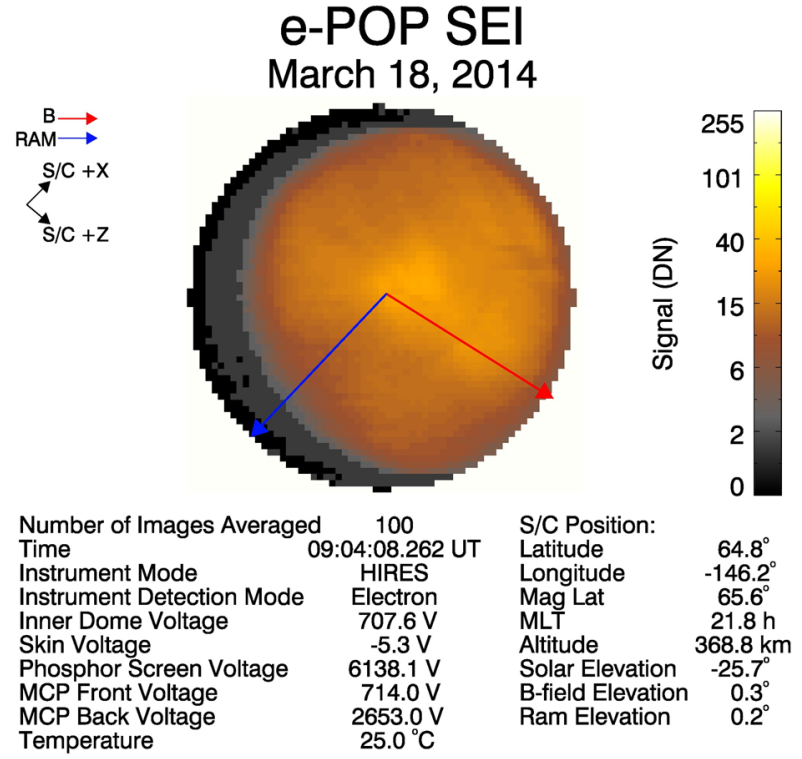

ePOP SEI - March 18, 2014
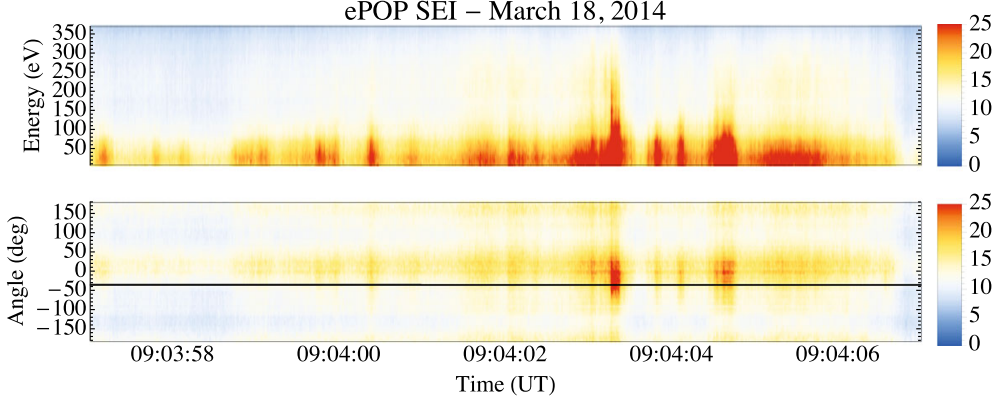

Fig. 10 Spectrograms of electron energy and detector angle (see Fig. 7) taken over a $10 \mathrm{~s}$ interval within a region of patchy pulsating aurora (same event as Fig. 9). The black solid line indicates the magnetic field direction. Intensity is measured in arbitrary (analog-to-digital converter) units

depends strongly on the detector gain, with contributions from both the MCPs and phosphor screen. Although gain values were characterized as a function of operating voltage prior to launch, a voltage monitor anomaly has hindered attempts at on-orbit calibration.

\section{Summary}

The e-POP SEI produces angle-energy images of low-energy charged particle distributions at a time resolution of $10 \mathrm{~ms}$. Through the use of a CCD-based detector, the instrument provides full coverage of the image plane with pixel density sufficient to resolve the finest features that can be produced by the electrostatic focusing system, leading to energy resolution $\Delta E / E$ of the order of $20 \%$, and azimuthal resolution of order $9^{\circ}$. Analog-mode operation of the CCD supports particle event rates that are orders of magnitude higher than typically achieved with particle-counting detectors, resulting in smaller statistical errors. 
Centroid calculations of rammed ion populations allows tracking of ion velocity variations to a resolution of $25 \mathrm{~m} / \mathrm{s}$ or better.

The e-POP SEI has been operating nominally on orbit since commissioning in late 2013. The SEI is most often operated in Hi-res mode, at maximum resolution in both time, at 100 images per second, and pixel density, as these two parameters represent what is most unique about the instrument. However, due to unanticipated telemetry restrictions, this allows the instrument to operate only 5-10 minutes per day, which is sufficient to cover an auroral zone crossing, for example. Normal mode (32-pixel diameter image) reduces telemetry enough to allow a continuous survey across the entire polar cap and auroral zones.

Despite the telemetry restrictions, careful planning of operations has created numerous scientific opportunities in conjunction with other e-POP instruments, as well as with groundbased facilities including cameras, radars, and ionospheric heaters. Potential collaborators are encouraged to contact the authors.

Acknowledgements The authors wish to thank the many individuals who contributed to the development and operation of the SEI, including Mark Astridge, Kaare Berg, Yi-Ching Chung, Troy Kachor, E. Peter King, Alexei Kouznetsov, Clifford Marcellus, Jamie Roberts, Ivan Wevers, and Andrew White. Thanks are also due to Magellan Bristol and MDA Corporation for their role in the development and flight of the SEI on e-POP, and to the Canadian Space Agency for funding and management of the e-POP project. J. Burchill was supported by a grant from the CSA; T. Cameron is an MSc student supported by a grant from the Natural Sciences and Engineering Research Council of Canada. Darren Chaddock provided the NASA THEMIS-ASI images used in support of Fig. 9. The THEMIS-ASI network is operated by UC Berkeley and the University of Calgary with contracts from NSF and CSA, respectively.

Open Access This article is distributed under the terms of the Creative Commons Attribution 4.0 International License (http://creativecommons.org/licenses/by/4.0/), which permits unrestricted use, distribution, and reproduction in any medium, provided you give appropriate credit to the original author(s) and the source, provide a link to the Creative Commons license, and indicate if changes were made.

\section{References}

J.G. Aase, J.P. Hackett, B. Moffat, J.K. Burchill, D.J. Knudsen, Spatial resolution and relative brightness of a microchannel plate detector system with P20 and P43 phosphor screens. Opt. Eng. 50(6), 064001 (2011)

T. Abe, B.A. Whalen, A.W. Yau, S. Watanabe, E. Sagawa, K.I. Oyama, Altitude profile of the polar wind velocity and its relationship to ionospheric conditions. Geophys. Res. Lett. 20, 2825-2828 (1993)

M. André, P. Norqvist, L. Andersson, L. Eliasson, A.I. Eriksson, L. Blomberg, R.E. Erlandson, J. Waldemark, Ion energization mechanisms at $1700 \mathrm{~km}$ in the auroral region. J. Geophys. Res. 103(A3), 4199-4222 (1998)

C.D. Anger, S.K. Babey, A.L. Broadfoot, R.G. Brown, L.L. Cogger, R. Gattinger, J.W. Haslett, R.A. King, D.J. McEwen, J.S. Murphree, E.H. Richardson, B.R. Sandel, K. Smith, A.V. Jones, An ultraviolet auroral imager for the Viking spacecraft. Geophys. Res. Lett. 14, 387-390 (1987)

H. Bahcivan, M.J. Nicolls, G. Perry, Comparison of SuperDARN irregularity drift measurements and F-region ion velocities from the resolute bay ISR. J. Atmos. Sol.-Terr. Phys. 105, 325-3311 (2013)

J.K. Burchill, GEODESIC observations of auroral ions. Ph. D. thesis, Univ. of Calgary, Calgary, Alberta, Canada (2003)

J.K. Burchill, D.J. Knudsen, B.J.J. Bock, R.F. Pfaff, D.D. Wallis, J.H. Clemmons, S.R. Bounds, H. StenbaekNielsen, Core ion interactions with BB ELF, lower hybrid, and Alfvén waves in the high-latitude topside ionosphere. J. Geophys. Res. 109(A1) (2004)

J.K. Burchill, D.J. Knudsen, J.H. Clemmons, K. Oksavik, R.F. Pfaff, C.T. Steigies, A.W. Yau, T.K. Yeoman, Thermal ion upflow in the cusp ionosphere and its dependence on soft electron energy flux. J. Geophys. Res. 115(A5) (2010)

J.K. Burchill, J.H. Clemmons, D.J. Knudsen, M. Larsen, M.J. Nicolls, R.F. Pfaff, D. Rowland, L. Sangalli, High-latitude E region ionosphere-thermosphere coupling: A comparative study using in situ and incoherent scatter radar observations. J. Geophys. Res. 117(A2) (2012) 
C.W. Carlson, D.W. Curtis, G. Paschmann, W. Michel, An instrument for rapidly measuring plasma distribution functions with high resolution. Adv. Space Res. 2(7), 67-70 (1982)

C.W. Carlson, R.F. Pfaff, J.G. Watzin, The fast auroral snapshot (FAST) mission. Geophys. Res. Lett. 25(12), 2013-2016 (1998)

L.L. Cogger et al., Ultraviolet auroral imager (UVAI), in Interball Mission and Payload, ed. by Y. Galperin, T. Muliarchik, J.P. Thouvenin (1995), p. 382. Russian Space Agency, Space Research Institute and French Space Agency

L.L. Cogger et al., The Fast Auroral Imager (FAI) for the e-POP MIssion. Space Sci. Rev. (2014). doi:10. 1007/s11214-014-0107-x

E. Donovan, S. Mende, B. Jackel, H. Frey, M. Syrjäsuo, I. Voronkov, M. Connors, The THEMIS all-sky imaging array-system design and initial results from the prototype imager. J. Atmos. Sol.-Terr. Phys. 68(13), 1472-1487 (2006)

E.H. Eberhart, An operational model for microchannel plate devices. IEEE Trans. Nucl. Sci. NS-28(1), 712 (1981)

S.A. Fuselier, A.G. Ghielmetti, T.E. Moore, M.R. Collier, J.M. Quinn, G.R. Wilson, P. Wurz et al., Ion outflow observed by IMAGE: Implications for source regions and heating mechanisms. Geophys. Res. Lett. 28, 1163-1166 (2001)

D.J. Knudsen, J.-E. Wahlund, Core ion flux bursts within solitary kinetic Alfvén waves. J. Geophys. Res. 103(A3), 4157-4169 (1998)

D.J. Knudsen, B.A. Whalen, A.W. Yau, M.J. Greffen, A.I. Eriksson, N. Lloyd, M. Boehm, J. Clemmons, L.G. Blomberg, Sub-kilometer thermal plasma structure near $1750 \mathrm{~km}$ altitude in the polar cusp/cleft. Geophys. Res. Lett. 21(17), 1907-1910 (1994)

D.J. Knudsen, J.H. Clemmons, J.-E. Wahlund, Correlation between core ion energization, suprathermal electron bursts, and broadband ELF plasma waves. J. Geophys. Res. 103(A3), 4171-4186 (1998)

D.J. Knudsen, J.K. Burchill, K. Berg, T. Cameron, G.A. Enno, C.G. Marcellus, E.P. King, I. Wevers, R.A. King, A low-energy charged particle distribution imager with a compact sensor for space applications. Rev. Sci. Instrum. 74(1), 202-211 (2003)

K.A. Lynch, R.L. Arnoldy, P.M. Kintner, P. Schuck, J.W. Bonnell, V. Coffey, Auroral ion acceleration from lower hybrid solitary structures: a summary of sounding rocket observations. J. Geophys. Res. 104(A12), 28515-28534 (1999). doi:10.1029/1999JA900289

E.A. MacDonald, K.A. Lynch, M. Widholm, R. Arnoldy, P.M. Kintner, E.M. Klatt, M. Samara, J. LaBelle, Giovanni Lapenta, In situ measurement of thermal electrons on the SIERRA nightside auroral sounding rocket. J. Geophys. Res. 111(A12) (2006)

W.K. Peterson, P.C. Chamberlin, T.N. Woods, P.G. Richards, Temporal and spectral variations of the photoelectron flux and solar irradiance during an X class solar flare. Geophys. Res. Lett. 35(12) (2008)

C.J. Pollock, T.E. Moore, M.L. Adrian, P.M. Kintner, R.L. Arnoldy, SCIFER: Cleft region thermal electron distribution functions. Geophys. Res. Lett. 23, 1881-1884 (1996)

L. Sangalli, D.J. Knudsen, M.F. Larsen, T. Zhan, R.F. Pfaff, D. Rowland, Rocket-based measurements of ion velocity, neutral wind, and electric field in the collisional transition region of the auroral ionosphere. J. Geophys. Res. 114(A4) (2009)

K. Stasiewicz et al., Small scale Alfvénic structure in the aurora. Space Sci. Rev. 92(3-4), 423-533 (2000)

R.J. Strangeway, R.E. Ergun, Y. Su, C.W. Carlson, R.C. Elphic, Factors controlling ionospheric outflows as observed at intermediate altitudes. J. Geophys. Res. 110(A3) (2005)

D.D. Wallis, D.M. Miles, B.B. Narod, J.R. Bennest, K.R. Murphy, I.R. Mann, A.W. Yau, The CASSIOPE/ePOP Magnetic Field Instrument (MGF). Space Sci. Rev. (2014). doi:10.1007/s11214-014-0105-Z

B.A. Whalen, D.J. Knudsen, A.W. Yau, A.M. Pilon, T.A. Cameron, J.F. Sebesta, D.J. McEwen et al., The Freja F3C cold plasma analyzer. Space Sci. Rev. 70.3-4, 541-561 (1994)

A.W. Yau, H.G. James, CASSIOPE e-POP Mission Overview. Space Sci. Rev. (2015). doi:10.1007/s11214015-0135-1

A.W. Yau et al., The e-POP IRM Instrument. Space Sci. Rev. (2014). doi:10.1007/s11214-015-0149-8 